\title{
THE VERY LITHIUM RICH POST-AGB SB2 BINARY HD 172481
}

To appear in the proceedings of the conference: "Post-AGB Objects (Proto-Planetary Nebulae) as a Phase of Stellar Evolution", held in Toruń, Poland, July 5-7, 2000, eds.

R. Szczerba, R. Tylenda, and S.K. Gorny.

Maarten Reyniers, Hans Van Winckel

Instituut voor Sterrenkunde, Celestijnenlaan 200B, 3001 Heverlee, Belgium

Maarten.Reyniers@ster.kuleuven.ac.be

\begin{abstract}
Double lined spectroscopic binaries in an evolved stage of evolution are expected to be extremely rare since they must consist of equally luminous and thus almost equally evolved objects, which requires an extremely similar initial mass. In this contribution we discuss such rare double evolved SB2 system: HD 172481. This binary includes an Ftype post-AGB object and an M-type AGB companion. The spectrum shows a surprisingly strong Li I $670.8 \mathrm{~nm}$ line with an equivalent width of $W_{\lambda}=54 \mathrm{~m} \AA$, yielding a lithium abundance of $\log \epsilon(\mathrm{Li})=3.6$. Several explanations for this huge lithium content are explored.
\end{abstract}

\section{HD 172481 IN THE POST-AGB SAMPLE}

In the course of our ongoing program to study the chemical composition of post-AGB stars (see Van Winckel, this volume), high resolution, high signal-to-noise spectra were taken of the F2Ia supergiant HD 172481. This star was previously selected as a candidate post-AGB star by Oudmaijer et al. (1992) because of its far infrared excess and its high galactic latitude $\left(b=-10.37^{\circ}\right)$.

\section{OBSERVATIONAL DATA}

Our photometric data consist of 54 data points in the Geneva photometric system between 1989 and 1996, near-IR JHKLM photometry taken with the ESO 1m telescope in 1992 and the IRAS-fluxes. 62 radial velocity measurements were obtained with the CORAVEL radial velocity spectrometer (Danish 1.5m telescope, La Silla, Chile) between 1983 and 1997 and 4 recent measurements with the CORALIE spectrograph (Euler telescope, La Silla, Chile). 5 high resolution, high signal-to- 
Figure 1 The 1998 EMMI (top) and FEROS (bottom) spectra in the $700-715 \mathrm{~nm}$ $\mathrm{TiO}$ band head region. The top spectrum is offset by 0.2 units. The TiO bands in the red spectrum of HD 172481 are a clear signature of its cool companion. Moreover, these bands vary in time as evidenced by our 1998 EMMI spectrum. Band head wavelengths are from Valenti et al. (1998).

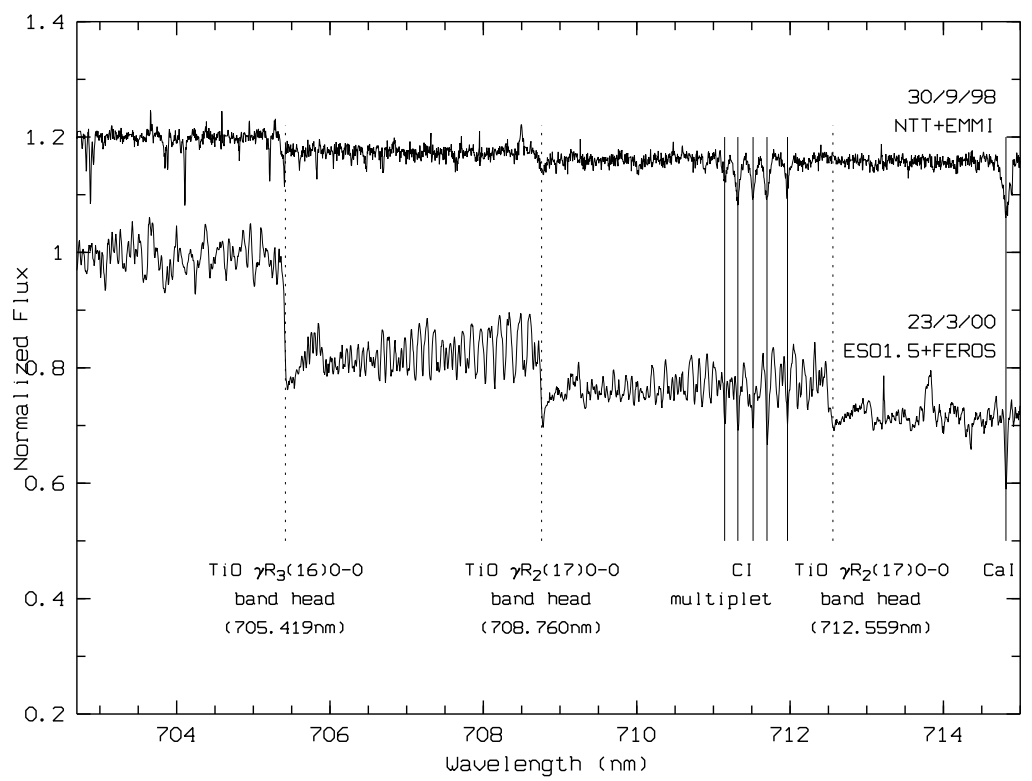

noise spectra were taken with several spectrographs and telescopes: 2 spectra with the UES (WHT, La Palma, Spain) in 1995, 2 with the EMMI spectrograph (NTT, La Silla, Chile) in 1997 and 1998 and 1 with FEROS (ESO 1.5m, La Silla, Chile) in 2000.

\section{HD172481 AS A BINARY}

A comparison of the blue and green regions of the spectra of this object with spectra of the normal massive F0Ib supergiant HR 1865 confirms the F2Ia0 spectral type given by the SIMBAD database. However, our 1997 EMMI and FEROS spectra show clear and strong discontinuous jumps at certain wavelengths in the red region which we identified as TiO band heads (Fig. 1). Since the pulsational amplitude is very low $(\Delta \mathrm{V} \sim 0.2)$, we conclude that these bands are caused by a cool M-type companion. The red region of the spectrum of HD 172481 is therefore a mixture of two spectral types: atomic lines induced by a hot component $(\sim 7250 \mathrm{~K})$ and $\mathrm{TiO}$ bands caused by a cool component $(\sim 3500 \mathrm{~K})$. Moreover, the strength of these bands is clearly variable in time (also Fig. 1). 
Figure 2 The dereddened SED of HD 172481 with a $\mathrm{E}(\mathrm{B}-\mathrm{V})=0.44$. The dotted line represents the Kurucz model with $\mathrm{T}_{\text {eff }}=7250 \mathrm{~K}$ and $\log (\mathrm{g})=1.5$; the dashed line the model with $\mathrm{T}_{\text {eff }}=3500 \mathrm{~K}$ and $\log (\mathrm{g})=0.5$; the full line is the combined model.

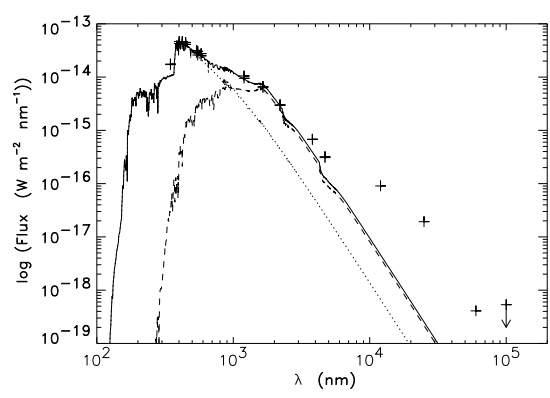

Figure 3 The abundances of HD 172481 relative to iron $[\mathrm{el} / \mathrm{Fe}]$. Errorbars are given if the number of lines is more than 3. The horizontal line represents $[\mathrm{el} / \mathrm{Fe}]=0$. Solar abundances are mainly taken from Grevesse (1989).

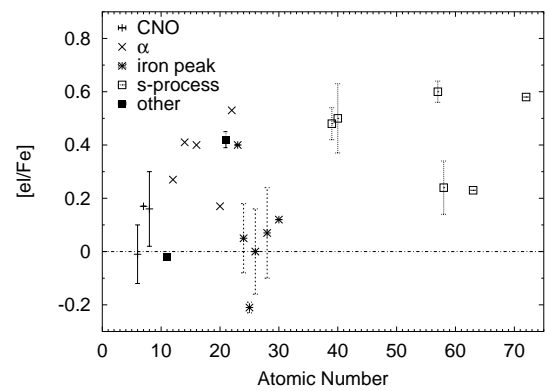

Other evidence confirming the binary nature of HD 172481 was found in its spectral energy distribution (SED hereafter), which is constructed from the collected photometry in Fig. 2 with an adopted reddening of $\mathrm{E}(\mathrm{B}-\mathrm{V})=0.44$. The fit of only the F-type (Kurucz) model atmosphere clearly did not satisfy from about $\lambda \sim 10^{3} \mathrm{~nm}$ redwards. Therefore, we had to invoke a second, cool component with $\mathrm{T}_{\text {eff }}=3500 \mathrm{~K}$. The latter temperature is, however, highly uncertain because it is derived by only a few photometric points.

Two more interesting remarks can be made concerning the SED. The luminosity ratio of both components $\mathrm{L}_{\mathrm{F}-\text { type }} / \mathrm{L}_{\mathrm{M} \text {-type }}$ is found to be 1.8. This ratio is rather dependent on the adopted reddening: using $\mathrm{E}(\mathrm{B}-$ $\mathrm{V})=0.2$, it equals to 1 . In any case, the two components must be of very similar luminosity. Consequently, also the M-type component must be very luminous and probably in its AGB phase of stellar evolution. A second remark concerns the IRAS-fluxes (the 4 most redward points on the SED) pointing to a cool infrared excess which we interpret as caused by a circumstellar dust shell or disk.

The binary nature of HD 172481 could, however, not be resolved by our radial velocity monitoring: although a peak-to-peak amplitude of $17 \mathrm{~km} \mathrm{~s}^{-1}$ is observed, we could not discover a long-term trend in the radial velocity data. A possible explanation for this fact can be an unfavourable inclination angle, but most likely it is due to a long orbital period. The variation induced by the orbital motion is then completely washed out by the (low amplitude) pulsation. This (semi-regular) lowamplitude pulsation in also seen in our Geneva photometry. 


\section{CHEMICAL ANALYSIS}

A detailed chemical abundance analysis was carried out based on the high resolution spectra. Atmospheric parameters were obtained with the commonly used spectroscopic method: excitation equilibrium for the effective temperature $\left(\mathrm{T}_{\text {eff }}\right)$, ionisation equilibrium for the gravity $(\log (g))$ and the microturbulent velocity $\left(\xi_{t}\right)$ is obtained by demanding that the iron abundance is independent of the reduced equivalent widths $\left(W_{\lambda} / \lambda\right)$ of the Fe I-lines. This method resulted in $\mathrm{T}_{\text {eff }}=7250( \pm 250) \mathrm{K}$, $\log (g)=1.5( \pm 0.5)(\operatorname{cgs})$ and $\xi_{t}=4( \pm 1) \mathrm{km} \mathrm{s}^{-1} .48 \mathrm{Fe} \mathrm{I}$ and $14 \mathrm{Fe}$ II lines were involved in this determination. We used the atmospheric models of Kurucz (CD-ROM set, 1993) in combination with his abundance calculation program WIDTH9. Our linelist contains only lines with well determined oscillator strengths in detail described in Van Winckel \& Reyniers (2000).

209 lines of 28 different ions were measured. We obtained a metallicity of $[\mathrm{Fe} / \mathrm{H}]=-0.55$. The results of our analysis are graphically presented in Fig. 3. On this figure, one can clearly see that

- the CNO elements are clearly not enhanced;

- the $\alpha$ elements are enhanced by $\sim 0.3$ to 0.4 dex, which is not an intrinsic enhancement, but a galactic evolution effect for this metallicity range;

- the other iron peak elements do follow the iron deficiency of $[\mathrm{Fe} / \mathrm{H}]=-0.55$;

- the light s-process elements $\mathrm{Y}$ and $\mathrm{Zr}$ and the heavy s-process element La are enhanced by $\sim 0.5$ dex.

\section{POST-AGB STATUS OF HD 172481}

In this section we collect our arguments for the proposed post-AGB status of HD 172481.

1. The high galactic latitude $\left(b=-10.37^{\circ}\right)$,

2. the large radial velocities $\left(<v>\sim-85 \mathrm{~km} \mathrm{~s}^{-1}\right)$ and

3 . the moderate metal deficiency $([\mathrm{Fe} / \mathrm{H}]=-0.55)$

point to a population II membership. Furthermore,

4. the SED (Fig. 2) shows the presence of dust, as a result of previous mass loss, probably during the AGB phase.

5. The photometry and the $\mathrm{H} \alpha$ profiles on our 4 spectra that include $\mathrm{H} \alpha$ show a variability very similar to other post-AGB stars ( $c f r$. Lèbre, this volume).

6. Finally, the slight s-process overabundances are probably the result of the third dredge-up, when He burning products are brought to the surface. This last argument is, however, not straightforward. If we expect the signature of a 3rd dredge-up, also the CNO elements should 
Figure 4 The LiI resonance line in HD 172481. left panel: The UES and FEROS spectrum around $671 \mathrm{~nm}$ with the Li I doublet of $54 \mathrm{~m} \AA$. The top spectrum is offset by 0.3 units. The contribution of the cool companion is larger for our FEROS run as evidenced by the detection of the (weak) TiO band head at $671.447 \mathrm{~nm}$ and the apparently lower $\mathrm{s} / \mathrm{n}$ for this spectrum. right panel: Synthesis of the LiI resonance line using our UES spectrum. A velocity broadening $(\zeta)$ of $14 \mathrm{~km} \mathrm{~s}^{-1}$ is used in this synthesis, deduced by the synthesis of unblended lines with a smooth profile. The points are the observed spectrum, the lines synthetic spectra with $\log \epsilon(\mathrm{Li})=3.47$, 3.57 and 3.67 resp. A least-squares fit resulted in $\log \epsilon(\mathrm{Li})=3.57$ (full line).
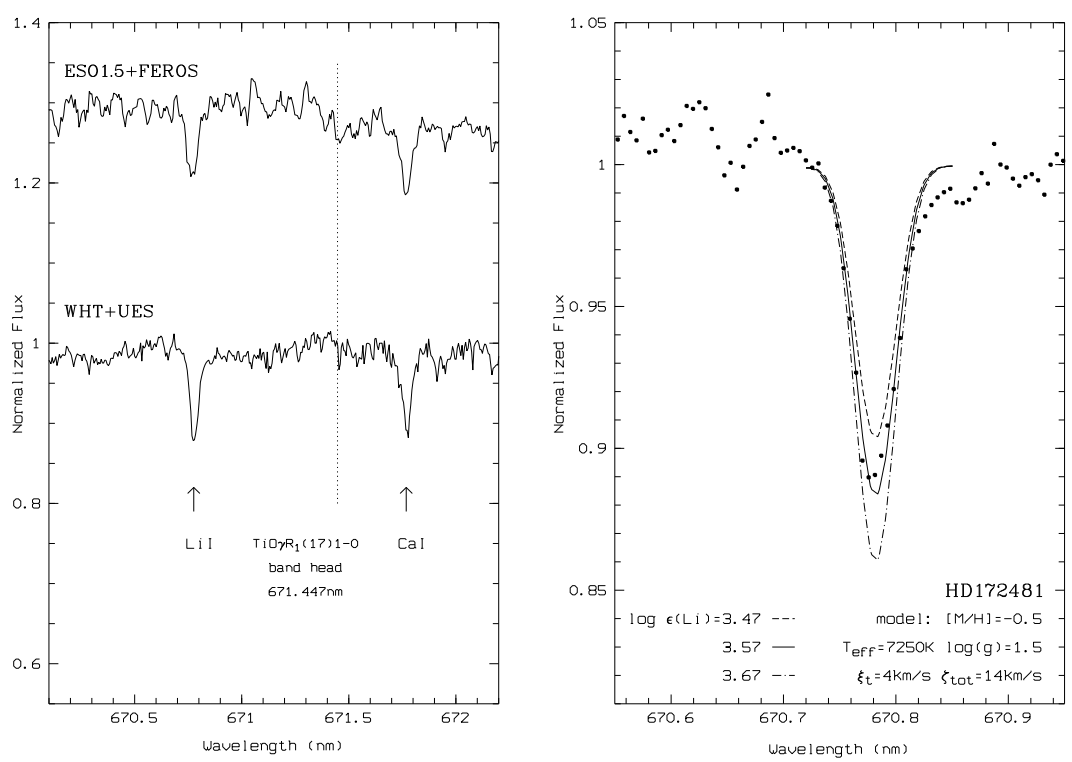

be enhanced, which is not detected: the total $\mathrm{CNO}$ abundance is equal to the initial value.

\section{LITHIUM IN HD 172481}

The most surprising discovery during our spectral analysis was the detection of a strong Li I resonance line at $670.8 \mathrm{~nm}$ (Fig. 4). Kurucz's synthesis program SYNTHE was used to account for the doublet structure of this line. A least-squares fit between the observed and the synthetic spectrum yielded an abundance of $\log (\mathrm{Li})=3.57 \pm 0.2$ (Fig. 4). It is far from clear how HD 172481 can be so Li rich, exceeding even the interstellar medium value of $\log \epsilon(\mathrm{Li})=3.3$. During the evolution of a star, lithium is destroyed, as it burns at relatively low temperatures. Lithium destruction and depletion is expected to occur during the mainsequence evolution (e.g. $\left.\log (\mathrm{Li})_{\odot}=1.16\right)$ as well as during the RGB and 
AGB evolution, as the deeper convective envelope reaches into lithiumdepleted interior layers.

Nevertheless Li rich evolved stars exist. In general three classes of Li rich evolved (AGB and post-AGB) stars can be distinguished: (1) the luminous "hot bottom burning" (HBB) S-type AGB stars in the Clouds (Plez et al. 1993, Smith et al. 1995) and the Galaxy (GarcíaLario et al. 1999); (2) galactic low-mass C stars (Abia et al. 1993; Abia \& Isern 1997, 2000) which are mostly J-type and (3) 4 metal-poor low-mass post-3rd dredge-up objects being IRAS 22272+5435 (Začs et al. 1995), IRAS 05341+0852 (Reddy et al. 1997), IRAS Z02229+6208 and IRAS $07430+1115$ (Reddy et al. 1999). Only for the HBB stars, $\mathrm{Li}$ production is supported by the models via the ${ }^{7} \mathrm{Be}$-transport mechanism (Cameron \& Fowler 1971). All models suggest that HBB and the associated Cameron-Fowler mechanism only works in intermediate mass stars (4-7 $\mathrm{M}_{\odot}$; e.g. Sackmann \& Boothroyd 1992).

For classes (2) and (3), which are most probably low-mass stars, no envelope burning is predicted in any AGB model. By its old nature, and hence low mass, a "hot bottom burning" scenario is not applicable to HD 172481. The most promising models to date, which could shed some light on the Li rich evolved low-mass stars are the "cool bottom processing" models by Wasserburg et al. (1995) and Sackmann \& Boothroyd (1999). These models are successfully developed to explain the Li rich K giants (e.g. de La Reza et al. 1997 and references therein) using a non-standard mixing mechanism. The same group of authors suggests that their models might also work in the TP-AGB phase of low-mass stars (Boothroyd \& Sackmann 1999).

\section{CONCLUSIONS}

In this contribution we shortly described our study on the very peculiar supergiant HD 172481. The TiO band heads superimposed on a typical F-type spectrum reveal that it is a binary object, which is further strengthened by the SED. The high radial velocities, the high galactic latitude and the moderate metal deficiency found in our chemical analysis suggest that the F-type component is an old and hence low-mass object. It is probably in its post-AGB phase of evolution as evidenced by the dust, the nature of the (low amplitude) variability and the slight s-process overabundances. Above all, this star is found to be super rich in lithium, with a $\mathrm{Li}$ abundance of $\log \epsilon(\mathrm{Li})=3.6$. A more detailed description can be found in Reyniers \& Van Winckel (2000). 


\section{Acknowledgments}

Both authors acknowledge financial support of the Fund for Scientific Research Flanders.

\section{References}

Abia C., Isern J., 1997, MNRAS 289, L11

Abia C., Isern J., 2000, ApJ 536, 438

Abia C., Boffin H.M.J., Isern J., Rebolo R., 1993, A\&A 272, 455

Boothroyd A.I., Sackmann J.-I., 1999, ApJ 510, 232

Cameron A.G.W., Fowler W.A., 1971, ApJ 164, 111

de La Reza R., Drake N.A., da Silva L., Torres C.A.O., Martin E.L., 1997, ApJ 482, L77

García-Lario P., D’Antona F., Lub J., Plez B., Habing H.J., 1999. In: Le Bertre T., Lèbre A., Waelkens C. (eds.) Proc. IAU Symp. 191, AGB Stars. ASP Conference Series, p. 91

Grevesse N., 1989. In: Waddington C.J. (ed.) AIP Conferences Series 183, Cosmic Abundances of Matter. American Institute of Physics, New York, p. 9

Oudmaijer R.D., van der Veen W.E.C.J., Waters L.B.F.M., et al., 1992, A\&ASS 96, 625

Plez B., Smith V.V., Lambert D.L., 1993, ApJ 418, 812

Reddy B.E., Parthasarathy M., Gonzalez G., Bakker E.J., 1997, A\&A 328, 331

Reddy B.E., Bakker E.J., Hrivnak B.J., 1999, ApJ 524, 831

Reyniers M., Van Winckel H., 2000, A\&A, in press (preprint on astro-ph/0010486)

Sackmann I.-J., Boothroyd A.I., 1992, ApJ 392, L71

Sackmann I.-J., Boothroyd A.I., 1999, ApJ 510, 217

Smith V.V., Plez B., Lambert D.L., Lubowich D.A., 1995, ApJ 441, 735

Valenti J.A., Piskunov N., Johns-Krull C.M., 1998, ApJ 498, 851

Van Winckel H., Reyniers M., 2000, A\&A 354, 135

Wasserburg G.J., Boothroyd A.I., Sackmann I.-J. 1995, ApJ 447, L37

Začs L., Klochkova V.G., Panchuk V.E., 1995, MNRAS 275, 764 